\title{
Static force characterization with Fano anti- resonance in levitated optomechanics
}

Cite as: Appl. Phys. Lett. 114, 023104 (2019); https://doi.org/10.1063/1.5081045

Submitted: 13 November 2018 . Accepted: 28 December 2018 . Published Online: 16 January 2019

Chris Timberlake, Marko Toroš ${ }^{(D)}$, David Hempston, George Winstone, Muddassar Rashid, and Hendrik Ulbricht
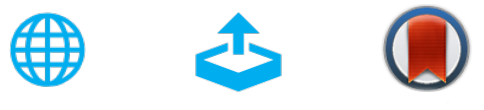

\section{ARTICLES YOU MAY BE INTERESTED IN}

Nanoscale thermal gradients activated by antenna-enhanced molecular absorption in the mid-infrared

Applied Physics Letters 114, 023105 (2019); https://doi.org/10.1063/1.5079488

AlN-based hybrid thin films with self-assembled plasmonic Au and Ag nanoinclusions Applied Physics Letters 114, 023103 (2019); https://doi.org/10.1063/1.5083950

Enhanced quantum dots spontaneous emission with metamaterial perfect absorbers Applied Physics Letters 114, 021103 (2019); https://doi.org/10.1063/1.5081688 


\title{
Static force characterization with Fano anti-resonance in levitated optomechanics
}

\author{
Cite as: Appl. Phys. Lett. 114, 023104 (2019); doi: 10.1063/1.5081045 \\ Submitted: 13 November 2018 - Accepted: 28 December 2018 • Published Online: \\ 16 January 2019
}

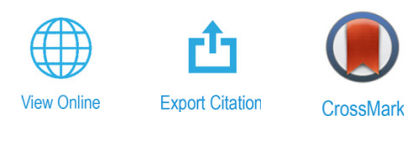

Chris Timberlake, ${ }^{1, a)}$ Marko Toroš, ${ }^{1, b)}$ (i) David Hempston, ${ }^{1}$ George Winstone, ${ }^{1,2}$ Muddassar Rashid, and Hendrik Ulbricht ${ }^{1, c}$ )

\author{
AFFILIATIONS \\ ${ }^{1}$ Department of Physics and Astronomy, University of Southampton, Southampton S017 1BJ, United Kingdom \\ ${ }^{2}$ School for Materials Science, Japan Advanced Institute of Science and Technology, Nomi, Ishikawa 923-1211, Japan \\ a) Electronic mail: ct10g12@soton.ac.uk \\ b) Electronic mail: m.toros@soton.ac.uk \\ c) Electronic mail: h.ulbricht@soton.ac.uk
}

\begin{abstract}
We demonstrate a classical analogy to the Fano anti-resonance in levitated optomechanics by applying a DC electric field. Specifically, we experimentally tune the Fano parameter by applying a DC voltage from $0 \mathrm{kV}$ to $10 \mathrm{kV}$ on a nearby charged needle tip. We find consistent results across negative and positive needle voltages, with the Fano line-shape feature able to exist at both higher and lower frequencies than the fundamental oscillator frequency. We can use the Fano parameter to characterize our system to be sensitive to static interactions which are ever-present. Currently, we can distinguish a static Coulomb force of $2.7 \pm 0.5 \times 10^{-15} \mathrm{~N}$ with the Fano parameter, which is measured with $1 \mathrm{~s}$ of integration time. Furthermore, we are able to extract the charge to mass ratio of the trapped nanoparticle.
\end{abstract}

Published under license by AIP Publishing. https://doi.org/10.1063/1.5081045

Resonance is of significant importance in a wide variety of fields within Physics, with the phenomena found in both classical and quantum systems. In 1961, Fano discovered that, in optics, an asymmetric line-shape arises by the interference between a discrete localized state and a continuum of states. ${ }^{1,2}$

Fano interference has been demonstrated in numerous quantum mechanical systems including semiconductor nanomaterials, ${ }^{3,4}$ quantum wells ${ }^{5,6}$ and quantum dots, ${ }^{7-9}$ superconductors, ${ }^{10,11}$ dielectric ${ }^{12}$ and gold nanoparticles, ${ }^{13}$ photonic crystals, ${ }^{14-20}$ electromagnetically induced transparency (EIT) in interactions between three-level atomic systems and two laser fields, ${ }^{21,22}$ and many other examples.

Although the Fano anti-resonance phenomenon has been widely acknowledged as an effect in quantum systems, it is a general wave phenomenon, meaning that it can also manifest itself in numerous classical systems. Studies on the classical interpretation of the Fano effect have been undertaken, ${ }^{23,24}$ as well as the theoretical comparison of the quantum and classical Fano parameters. ${ }^{25}$ Experimental evidence of Fano anti-resonance has been shown for classical nanomechanical oscillators, ${ }^{26-30}$ whispering-gallery microresonators, ${ }^{31}$ and prism-coupled square micro-pillars.
Levitated nanoparticles have recently emerged as very promising candidates for measuring extremely small forces. This is typically done by measuring the resonant response to a perturbation on its motion. ${ }^{33-35}$ Levitated systems have also been used to study interactions with nearby dielectric surfaces, ${ }^{36,37}$ and proposals have been devised to measure short range interactions such as the Casimir effect. ${ }^{38}$ Recently, Hebestreit et al. proposed and demonstrated the detection of static forces using free falling nanoparticles, with a sensitivity of $10 \mathrm{aN}$ reported. ${ }^{39}$

In this letter, we experimentally demonstrate Fano antiresonance in levitated optomechanics. The characteristic Fano anti-resonance is induced with a static Coulomb interaction by charging a stainless steel needle close to a charged nanoparticle in a gradient force optical trap. We show that we can tune the Fano parameter by varying the voltage applied to the needle tip and use the asymmetry in the line-shape to characterize a method of static force detection. The advantage of this method is precise force sensing irrespective of the resonant frequency. The consistent Fano parameter results were found for positive and negative applied voltages. In addition, we are able to extract the charge to mass ratio of the trapped nanoparticle. We also 
give a phenomenological model to describe the Fano line-shape and extract a characteristic rate.

We consider a polarizable nanoparticle trapped in an optical trap which has been described in Ref. 40. It is convenient to define the center of the optical trap as the origin of the coordinate system: the $x$ axis is the vertical direction pointing away from the ground, the $z$ axis is oriented in the direction of the beam propagation away from the mirror, and the $y$ axis is the remaining horizontal axis (see Fig. 1). To stabilize the motion at low pressure $p$, the nanoparticle is cooled using parametric feedback cooling. ${ }^{41-43}$ The strong cooling confines the motion of the nanoparticle to small oscillations, $\delta \boldsymbol{r}=(\delta x, \delta y, \delta z)^{\top}$, around an equilibrium position, $\boldsymbol{r}_{\text {eq. }}=\left(x_{0}, y_{0}, z_{0}\right)^{\top}$, which effectively decouples the translational motions. As we will discuss below, the scattering force $\mathbf{F}_{\mathrm{s}}=\left(0,0, \mathrm{~F}_{\text {scatt }}\right)$ displaces the equilibrium point $\boldsymbol{r}_{\text {eq. }}$ away from the mirror, i.e., $x_{0}, y_{0} \ll z_{0}$, which makes the $z$-dynamics independent of the $x_{0}$ and $y_{0}$ values. In the following, we limit the discussion to the $z$-motion as it is experimentally the strongest signal.

The potential generated by the optical field can be modeled using the following potential:

$$
\mathrm{U}_{\mathrm{opt}}(z)=\frac{m}{2} \omega_{0}^{2} z^{2}-\eta z^{4},
$$

where $m$ is the mass of the nanoparticle, $\omega_{0}^{2}=\frac{2 \mathrm{P} \%}{c \sigma_{L} \rho z^{2}},{ }^{44} \mathrm{P}$ is the laser power, $\sigma_{\mathrm{L}}=\pi w_{0}^{2}$ is the effective laser beam cross sectional area, $w_{0}$ is the mean beam waist radius, $z_{\mathrm{R}}$ is the Rayleigh length, $\rho$ is the particle density, $\chi$ is an electric susceptibility of the particle, $\mathrm{c}$ is the speed of light, and $\eta$ quantifies the dominant nonlinearity of the trap. ${ }^{45}$

The scattering force has only one non-zero component $F_{\text {scatt }}(z)$ which is given in Ref. 46. We integrate it, i.e., $\int d z^{\prime} \mathrm{F}_{\text {scatt }}\left(z^{\prime}\right)$, which gives the following effective potential:

$$
\mathrm{U}_{\text {scatt }}(z)=-\frac{32 \pi^{3} \hbar \Gamma_{\mathrm{s}} \mathrm{w}_{0}^{2} \tan ^{-1}\left(\frac{\lambda \mathrm{z}}{\pi \mathrm{w}_{0}^{2}}\right)}{3 \lambda^{2}},
$$

where $\Gamma_{S}=\frac{\sigma_{R}}{\sigma_{L}} \frac{P}{h \omega_{L}}$ is the scattering rate, $\sigma_{R}=\frac{\pi^{2} V_{0}^{2}}{\gamma^{4}}$ is an effective cross-sectional area, $\omega_{\mathrm{L}}=\frac{2 \pi \mathrm{c}}{\lambda}$, and $\lambda$ is the wavelength of light.

Consider now also a nearby charged needle which generates a Coulomb potential $\propto 1 /|\mathbf{R}-\boldsymbol{r}|$, where $\mathbf{R}=\left(\frac{1}{\sqrt{2}} R, 0, \frac{1}{\sqrt{2}} R\right)$ is the position of the needle tip. For the experimental situation

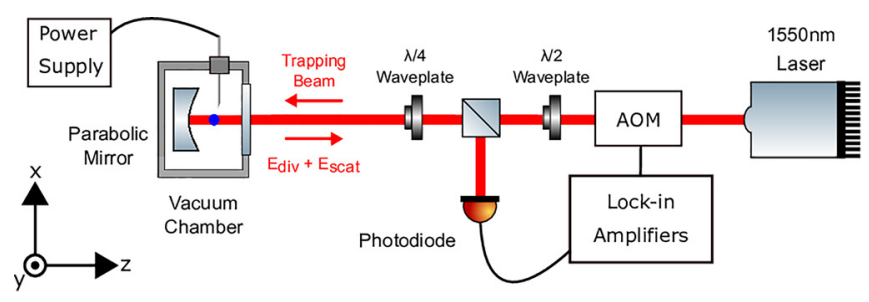

FIG. 1. Experimental setup. A silica nanoparticle is trapped and detected, with a $1550 \mathrm{~nm}$ laser at the focus of a paraboloidal mirror. An AOM is used to modulate the laser power and cool the motion of the nanoparticle. A nearby stainless steel needle is connected to a power supply to apply a voltage which is used to manipulate the motion of a charged nanoparticle in the trap. described in this paper, only the linear contribution is relevant; specifically, expanding the Coulomb potential to and including order $\mathcal{O}(z)$, one readily finds

$$
\mathrm{U}_{\mathrm{el}}(z)=\frac{q \mathrm{Q} z}{4 \pi \epsilon_{0} \sqrt{2} \mathrm{R}^{2}},
$$

where $Q$ is the charge on the needle tip, $q$ is the charge on the nanoparticle, and $\epsilon_{0}$ is the permittivity of free space.

In addition, we apply sinusoidal modulations of the laser power P to cool the center-of-mass motion (c.m.) of the nanonparticle, namely, parametric feedback cooling. ${ }^{41-43}$ In a nutshell, one can cool a translational degree of freedom by tracking its phase (in classical phase space) and applying a modulation at twice its harmonic frequency. Specifically, the feedback term can be obtained by making the formal replacement $\mathrm{P} \rightarrow \mathrm{P}\left(1+\beta z p_{z}\right)$ in the equations of motion, where $p_{z}$ denotes the conjugate momentum and $\beta$ is the strength of the feedback which has units of the inverse of an action, i.e., $\mathrm{kg}^{-1} \mathrm{~m}^{-2} \mathrm{~s}$. This procedure generates the following feedback force term in the dynamics: ${ }^{43}$

$$
f_{\mathrm{fb}}=\beta \partial_{z}\left(\mathrm{U}_{\mathrm{opt}}+\mathrm{U}_{\mathrm{scatt}}\right) z p_{z} .
$$

Taking into account the feedback term, one obtains the following dynamics:

$$
\begin{gathered}
\dot{z}=\frac{z}{m}, \\
\dot{p}_{z}=-\partial_{z} \mathrm{U}_{\text {eff }}-f_{\mathrm{fb}}-2 \gamma_{\text {coll }} p_{z}+m \xi,
\end{gathered}
$$

where the total effective potential is given by $U_{\text {eff }}=U_{\text {opt }}$ $+U_{\text {scatt }}+U_{\text {el }}$. We have also included a damping term with coupling $\gamma_{\text {coll }}$, which is the gas collision rate, and a noise term $\xi$.

We now combine Eqs. (5) and (6), using $\dot{p}_{z}=m \dot{z}$, and Taylor expansion around the minimum position $z_{0}$, up to and including order $\mathcal{O}\left(\delta z^{2}\right)$. Specifically, we obtain the term $m \omega_{\mathrm{m}}^{2} \delta z^{2} / 2$, where the harmonic frequency is given by

$$
\omega_{\mathrm{m}}=\sqrt{\omega_{0}^{2}-\eta \frac{12 \mathrm{z}_{0}^{2}}{\mathrm{~m}}+\frac{64 \pi^{4} \lambda \mathrm{w}_{0}^{4} \hbar \mathrm{z}_{0}}{3 \mathrm{~m}\left(\pi^{2} \mathrm{w}_{0}^{4}+\lambda^{2} \mathrm{z}_{0}^{2}\right)^{2}} \Gamma_{\mathrm{s}} .}
$$

Equation (7) describes how the measured frequency $\omega_{\mathrm{m}}$ is related to the dipole-trap harmonic frequency $\omega_{0}$, the trap nonlinearity term $\propto \eta$, and the scattering force term $\propto \Gamma_{s}$. Here, we neglect other smaller effects that could change the particle's frequency.

We are left to discuss the noise term $\xi$. Suppose that the noise is invariant under time-translations, has zero mean, and is fully quantified by the two point correlation function $f(\tau)=\mathbb{E}[\xi(t+\tau) \xi(t)]$, where $\mathbb{E}[\cdot]$ denotes the average over different noise realizations. Exploiting the Wiener-Khinchin theorem, ${ }^{47}$ one then readily finds the power spectral density of the $z$-degree of freedom: $S_{z z}(\omega) \propto \tilde{f}(\omega) /\left(\omega^{2} \Gamma^{2}+\left(\omega^{2}-\omega_{\mathrm{m}}^{2}\right)^{2}\right)$, where $\tilde{f}$ denotes the Fourier transform of $f(\tau)$ and $\Gamma$ is an effective damping rate comprising $\gamma_{\text {coll }}$ and an additional damping contribution due to the feedback term. Furthermore, assume that $\tilde{f}$ has two distinct noise sources: one related to gas collisions $\propto \gamma_{\text {coll }}$ and the other related to the Coulomb force $\propto q \mathrm{Q}$. The term related to gas collisions, which is commonly present in 
optomechanical systems, leads to the usual Lorentzian power spectral density

$$
\mathrm{S}_{\text {coll }}(\omega) \propto \frac{\gamma_{\text {coll }}}{\omega^{2} \Gamma^{2}+\left(\omega^{2}-\omega_{\mathrm{m}}^{2}\right)^{2}} .
$$

Here, we also find a noise source $\propto q Q$. Here, we limit the analysis to a phenomenological description of the effect, leaving a proper derivation for future work. We make the following ansatz for the power spectral density associated with the noise perturbed Coulomb force:

$$
\mathrm{S}_{\mathrm{el}}(\omega) \propto \frac{\left(-\mathfrak{f} \gamma_{\mathrm{el}}^{2}+\left(\omega^{2}-\omega_{\mathrm{m}}^{2}\right)\right)^{2}}{\omega^{2} \Gamma^{2}+\left(\omega^{2}-\omega_{\mathrm{m}}^{2}\right)^{2}},
$$

where $\mathfrak{f}= \pm e_{0}^{2} /(q \mathrm{Q})$ is a number, $e_{0}$ denotes the unit charge of one electron, and $\gamma_{\mathrm{el}}$ is a characteristic rate. This ansatz has been inspired by the asymmetric Fano line-shape, ${ }^{1}$ and we will refer to $f$ as the Fano parameter. We speculate that $S_{\text {el }}(\omega)$ could originate from a non-Markovian noise, self-induced by the motion of the nanoparticle, i.e., the motion of the nanoparticle could be perturbing the charges on the needle tip and on the particle, which would perturb back the motion of the nanoparticle on a time scale $\propto \mathfrak{f}^{-1 / 2} \gamma_{\mathrm{el}}^{-1}$. Another possibility is that the noise could originate from vibrations in the silica nanoparticle. ${ }^{48}$

Adding all the contributions from Eqs. (8) and (9), we finally arrive at

$$
\mathrm{S}_{z z}(\omega)=\mathrm{A}+\mathrm{BS}_{\mathrm{coll}}(\omega)+\mathrm{CS}_{\mathrm{el}}(\omega),
$$

where $\mathrm{A}, \mathrm{B}$, and $\mathrm{C}$ are free parameters, i.e., fitting constants which account for the finite noise floor and normalizations. The order of magnitude of $A$ and $B$ can be first fixed when the charge on the needle is absent. This leaves two free parameters, $C$ and $\gamma_{\mathrm{el}}$.

The experimental setup consists of an optical gradient force trap, which is generated by tightly focusing a $1550 \mathrm{~nm}$ laser with a high numerical aperture (N.A.) paraboloidal mirror. ${ }^{35,42,49}$ Silica nanoparticles of radius $\sim 75 \mathrm{~nm}\left(\right.$ density $\left.\sim 1800 \mathrm{~kg} / \mathrm{m}^{3}\right)$ are prepared in a water based solution before being dispersed into a vacuum chamber, where a single particle is trapped at the focus. The position of the trapped nanoparticle is measured by detecting the interference generated between the Rayleigh scattered light from the particle, $E_{\text {scat }}$, and the divergent reference field, $E_{\text {div }}$, at a single photodiode (as shown in Fig. 1). The detected signal consists of information of three distinct translational frequency modes, which we refer to as $x, y$, and $z$. These three modes are tracked using lock-in amplifiers, which feed the information to an acousto-optic modulator (AOM) which applies a modulation to the laser intensity to parametrically cool the c.m. motion of the nanoparticle. A stainless steel needle, which is connected to a high voltage power supply, is placed close to the trapping region allowing us to influence the motion of a charged nanoparticle via the Coulomb interaction. The fine tip of the needle results in the charge being concentrated on the needle tip, meaning that the charged needle can be considered to be a point charge. ${ }^{3}$

The voltage applied to the needle is swept from $0 \mathrm{kV}$ to $10 \mathrm{kV}$, with $1 \mathrm{~s}$ of data taken for each voltage interval, and the effect this has on the shape of the $z$ frequency spectrum is

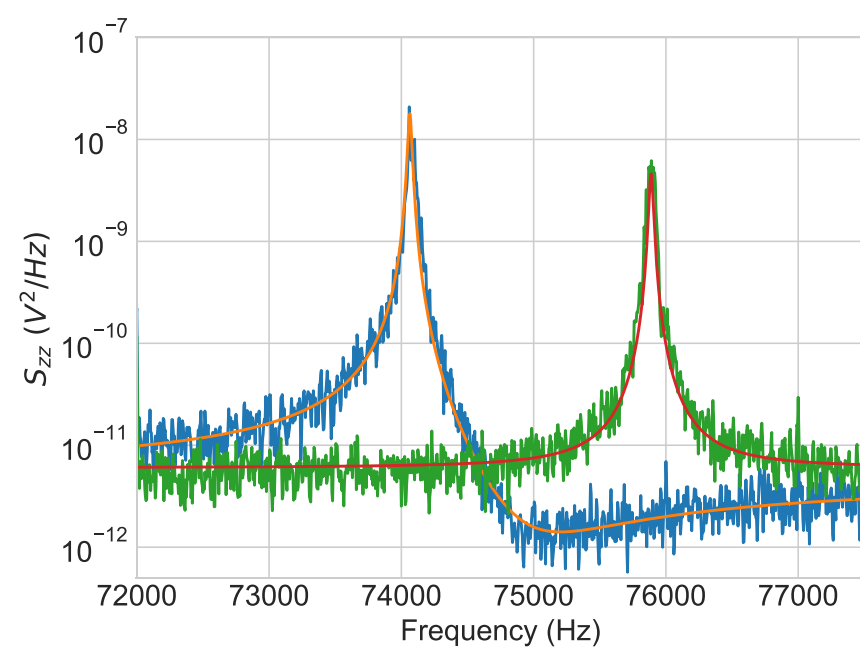

FIG. 2. The power spectral density of a levitated nanoparticle with $0 \mathrm{kV}$ applied to the needle (green) and $10 \mathrm{kV}$ (blue). The $0 \mathrm{kV}$ peak is fitted with a standard Lorentzian distribution from Eq. (8) while the $10 \mathrm{kV}$ peak is fitted with the asymmetric Fano line-shape from Eq. (10).

shown in Fig. 2. Due to the anti-resonance suppressing the mechanical noise, there is also a reduction in the noise floor compared to the standard Lorentzian; for an applied voltage of $10 \mathrm{kV}$, this reduction is approximately a factor of five for a small bandwidth around $1 \mathrm{kHz}$ from the $z$ frequency.

We have fitted Eq. (10) to data for two trapped particles in Fig. 3, exploring the regime of both the positive and negative Fano parameters, for which we find an average characteristic rate, $\bar{\gamma}_{\mathrm{el}}$, of $3.2 \pm 0.3 \mathrm{GHz}$ (positive Fano parameter) and $3.7 \pm 0.8 \mathrm{GHz}$ (negative Fano parameter), respectively. We find excellent agreement at low voltages $(<5 \mathrm{kV})$, with a slight

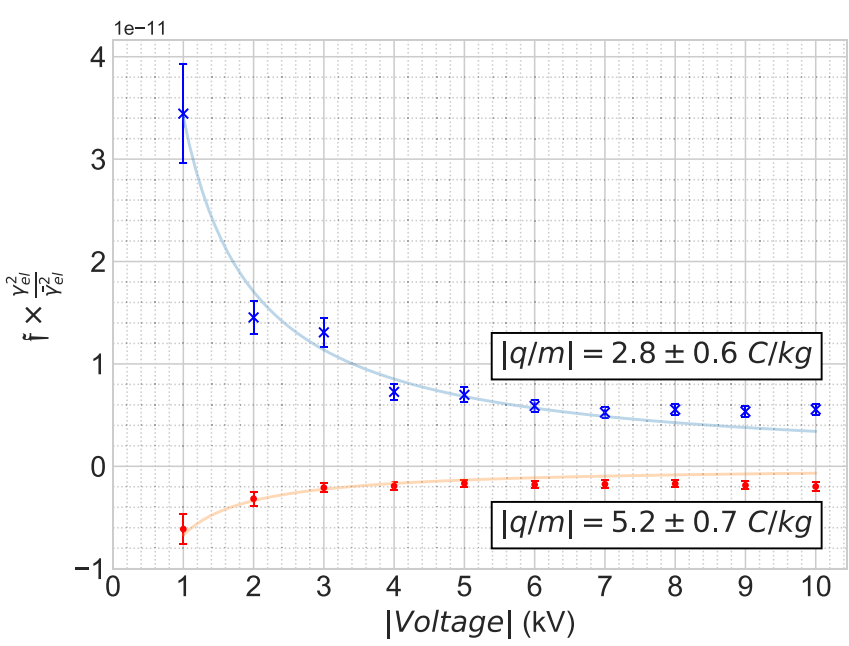

FIG. 3. The Fano parameter $f$ for two particles as a function of applied voltage, extracted using Eq. (10). The data were taken at a vacuum pressure of $8 \times 10^{-5}$ mbar and $3 \times 10^{-5}$ mbar for the blue and red data, respectively. The Fano parameter $f$ scales with the inverse of voltage, which is in good agreement with the theory. 
deviation at higher voltages $(>5 \mathrm{kV})$ which might be due to additional noise sources such as the ripple voltage in the high voltage power supply.

The application of an electric field also results in a shift in the average position $z_{0}$. Specifically, in the experimentally considered regime, we find an approximate expression for the minimum position $z_{0} \approx-\frac{1}{m \omega_{0}^{2}}\left(\frac{q Q}{4 \pi \epsilon_{0} \sqrt{2} R^{2}}-\frac{32 \pi^{2} \Gamma_{s} \hbar}{3 \lambda}\right)$. Furthermore, as the laser intensity is different in the new equilibrium position $z_{0}$, there is also a corresponding shift in oscillation frequency $\omega_{\mathrm{m}}$. This frequency shift is generally highly dependent on both the scattering force and the non-linearities in the gradient force potential, ${ }^{45}$ as can be seen in Eq. (7). However, limiting again the discussion to the regime where non-linearities can be neglected, we find a simplified expression for the frequency shift $\omega_{\mathrm{m}} \approx \omega_{0}\left(1+\frac{32 \Gamma_{\mathrm{S}} \lambda \hbar}{3 w_{0}^{4}} \frac{z_{0}}{m \omega_{0}^{2}}\right)$, where $\omega_{0}$ is given in Eq. (1). Combining the formulas in this paragraph and exploiting the formula for the scattering rate $\Gamma_{\mathrm{S}} \propto \mathrm{m}^{2}$ in Eq. (2), we obtain the following simplified expression for the mechanical frequency:

$$
\omega_{\mathrm{m}}=\omega_{0}+\mathcal{B} m^{2}+\mathcal{C} q \mathrm{Q},
$$

where $\mathcal{B}=\left(\frac{16 \pi}{3} \frac{P}{\omega_{0}^{3 / 2} w_{0}^{4} c \lambda^{3} \rho^{2}}\right)^{2}$ and $\mathcal{C}=\frac{2 \sqrt{2} P}{3 \pi \epsilon_{0} R^{2} \lambda^{2} w_{0}^{6} \omega_{0}^{3} \rho^{2} c}$ depend only on the trapping laser field, the optics, and the intrinsic particle properties, i.e., density $\rho$ and the electric susceptibility $\chi$; all these parameters can be estimated independently. Furthermore, we can also estimate the charge Q on the needle tip from the applied voltage V using COMSOL Multiphysics; we have tested the reliability of the numerical simulation by comparing with analytical methods used in previous works, ${ }^{35}$ and since we can set the desired voltage to high precision, we can in the first approximation neglect the error on the estimated value of Q. By fitting Eq. (11), we can then extract the values of the mass $m$ and charge $q$. The former value, $m$, is in good agreement with the mass we obtain by fitting to the frequency spectrum of the particle. ${ }^{41,42}$ From the values of $q$ and $m$, we can then estimate the charge to mass ratio $\mathrm{q} / \mathrm{m}$. The frequency shift due to the varying voltages for two different particles is shown in Fig. 4.

Static perturbations which cannot be "switched off" are typically hard to characterize because the oscillator experiences this static effect at all times, whereas the Fano parameter induced by such a perturbation will be present to allow characterization without "switching off," or varying, such perturbation. For example, forces such as those induced by radiation pressure or the Earth's gravitational attraction could be probed using this technique. Here, we use the Coulomb interaction to characterize how sensitive our system is to such static fields.

From the experimental data in Fig. 4, we can also extract the magnitude of the Coulomb force by fitting again Eq. (11) as discussed above: here, we are interested only in the extracted value of the charge product, $q \mathrm{Q}$. We can then obtain the Coulomb force given by $F=\frac{1}{4 \pi \epsilon_{0}} \frac{q Q}{R^{2}}$, as we can measure $R$ independently. In the following, we discuss the results for a particle of radius $71 \pm 11 \mathrm{~nm}$, which has a charge of $|q|=48 \pm 9 e_{0}$ (charge to mass ratio $|\mathrm{q} / \mathrm{m}|=2.8 \pm 0.6 \mathrm{C} / \mathrm{kg}$ ). For a $1 \mathrm{kV}$ applied voltage, we find $F=2.7 \pm 0.5 \times 10^{-15} \mathrm{~N}$. In principle, much smaller forces could be detected, which warrants further experimental

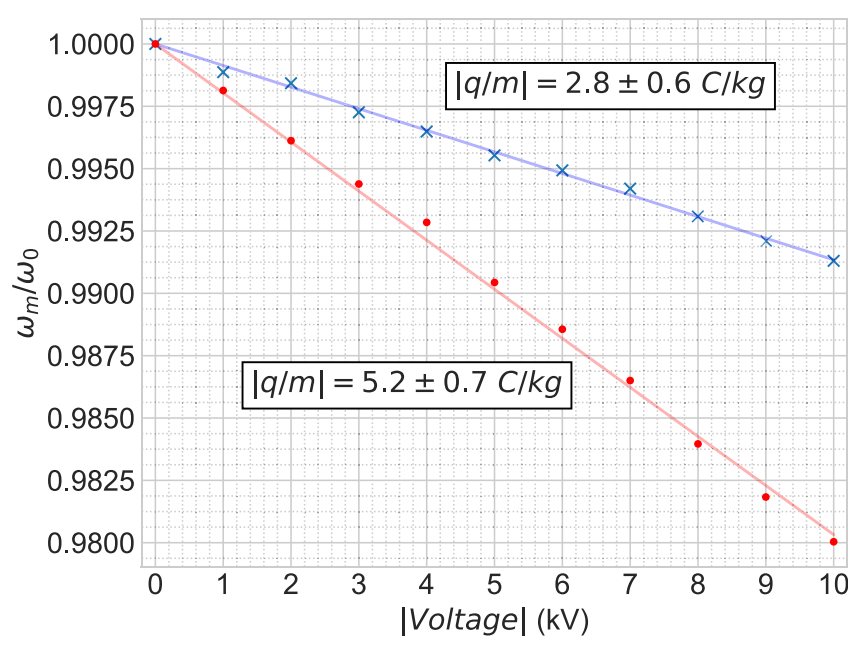

FIG. 4. Frequency shift of the $z$ motion as a function of applied voltage to the needle, fitted using Eq. (7). The applied voltage displaces the equilibrium position of the particle's motion, which changes the total potential $U_{\text {eff }}$ the particle experiences. This results in a frequency shift which is linear with the increasing voltage, to first approximation. Here, two particles are plotted to show the linear response to the applied voltage. It can be seen that a larger charge to mass ratio results in a larger relative frequency shift.

investigation. For more sensitive characterization of static perturbations, further data at $<1 \mathrm{kV}$ voltage on the needle are needed (or a smaller particle charge).

The needle setup can also be used for electrical state-based feedback control, such as cooling, of the motion of a charged nanoparticle. ${ }^{50,51}$ A single needle can be used to drive or cool the motion of the three translational degrees of freedom. However, by adding further two needles, each degree of freedom could be controlled independently by using the combination of the three fields to have our electric field vector pointing in any desired direction. Currently, we implement parametric feedback control by modulating the power of the trapping laser. The same laser is used for detection, meaning that the feedback signal is encoded in the detected signal. In principle, the needle setup is advantageous as it allows us to implement linear feedback at the oscillator frequency without mixing such a signal with the particle's detected motion. Similar feedback schemes have been used in cavity optomechanics and cantilever systems, where the modulation of radiation pressure ${ }^{52-54}$ and ac magnetic fields ${ }^{55}$ has been used to cool the oscillator mode.

In conclusion, we have experimentally demonstrated Fano anti-resonance in levitated optomechanics by introducing an electrostatic perturbation to a charged levitated nanoparticle's potential, with the ability to tune the Fano parameter by varying the applied voltage on a nearby charged needle. We have experimentally extracted the charge to mass ratio of trapped nanoparticles. Furthermore, we have shown that we can use the induced Fano parameter as a tool to quantify static interactions which perturb the nanoparticle's trapping potential, with a static detectable force of $2.7 \pm 0.5 \times 10^{-15} \mathrm{~N}$ reported. Although our results are not yet comparable to the $10 \mathrm{aN}$ static force sensitivity reported elsewhere, ${ }^{39}$ we note that our method can measure 
the force in $1 \mathrm{~s}$, compared to averaging the results of thousands of free fall experiments. As a sensor, this could be used as a tool to measure short range interactions, such as the Casimir force, or for sensitive gravity detection. The source of $\gamma_{\mathrm{el}}$ is currently unknown, but it could be due to vibrational phonons inside the silica nanoparticle. ${ }^{48}$

We would like to acknowledge A. Setter, A. Vinante, and L. Ferialdi for discussions and G. Savage and P. Connell for their technical expertise. We would also like to thank the Leverhulme Trust (RPG-2016-046) and the EU Horizon 2020 research and innovation programme under Grant Agreement No. 766900 [TEQ] for funding support. All data supporting this study are openly available from the University of Southampton repository at https://doi.org/10.5258/SOTON/D0713.

\section{REFERENCES}

${ }^{1} U$. Fano, Phys. Rev. 124, 1866 (1961).

${ }^{2}$ U. Fano, Il Nuovo Cimento 12, 154 (1935).

${ }^{3}$ C. P. Holfeld, F. Löser, M. Sudzius, K. Leo, D. M. Whittaker, and K. Köhler, Phys. Rev. Lett. 81, 874 (1998).

${ }^{4}$ P. Fan, Z. Yu, S. Fan, and M. L. Brongersma, Nat. Mater. 13, 471 (2014).

${ }^{5}$ J. Faist, F. Capasso, C. Sirtori, K. W. West, and L. N. Pfeiffer, Nature 390, 589 (1997).

${ }^{6}$ D. E. Nikonov, A. Imamoglu, and M. O. Scully, Phys. Rev. B 59, 12212 (1999).

${ }^{7}$ K. Kobayashi, H. Aikawa, S. Katsumoto, and Y. Iye, Phys. Rev. Lett. 88, 256806 (2002).

${ }^{8}$ A. C. Johnson, C. M. Marcus, M. P. Hanson, and A. C. Gossard, Phys. Rev. Lett. 93, 106803 (2004)

${ }^{9}$ S. Sasaki, H. Tamura, T. Akazaki, and T. Fujisawa, Phys. Rev. Lett. 103 266806 (2009).

${ }^{10}$ M. Limonov, A. Rykov, S. Tajima, and A. Yamanaka, Phys. Rev. Lett. 80, 825 (1998).

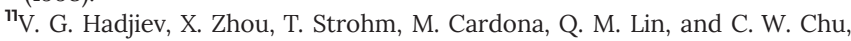
Phys. Rev. B 58, 1043 (1998).

${ }^{12}$ K. E. Chong, B. Hopkins, I. Staude, A. E. Miroshnichenko, J. Dominguez, M. Decker, D. N. Neshev, I. Brener, and Y. S. Kivshar, Small 10, 1985 (2014).

${ }^{13}$ M. I. Stockman, Nature 467, 541 (2010).

${ }^{14}$ A. E. Miroshnichenko, S. Flach, and Y. S. Kivshar, Rev. Mod. Phys. 82, 2257 (2010).

${ }^{15}$ I. V. Soboleva, V. V. Moskalenko, and A. A. Fedyanin, Phys. Rev. Lett. 108, 123901 (2012).

${ }^{16}$ H. Yang, D. Zhao, S. Chuwongin, J.-H. Seo, W. Yang, Y. Shuai, J. Berggren, M. Hammar, Z. Ma, and W. Zhou, Nat. Photonics 6, 615 (2012).

${ }^{17}$ M. Rybin, A. Khanikaev, M. Inoue, A. Samusev, M. Steel, G. Yushin, and M. Limonov, Photonics Nanostruct.-Fundam. Appl. 8, 86 (2010).

${ }^{18}$ W. Zhou, D. Zhao, Y.-C. Shuai, H. Yang, S. Chuwongin, A. Chadha, J.-H. Seo, K. X. Wang, V. Liu, Z. Ma, and S. Fan, Prog. Quantum Electron. 38, 1 (2014).

${ }^{19}$ P. Markoš, Phys. Rev. A 92, 043814 (2015).

${ }^{20}$ M. F. Limonov, M. V. Rybin, A. N. Poddubny, and Y. S. Kivshar, Nat. Photonics 11, 543 (2017).

${ }^{21}$ J. P. Marangos, J. Mod. Opt. 45, 471 (1998).

${ }^{22}$ M. Fleischhauer, A. Imamoglu, and J. P. Marangos, Rev. Mod. Phys. 77, 633 (2005).

${ }^{23}$ Y. S. Joe, A. M. Satanin, and C. S. Kim, Phys. Scr. 74, 259 (2006).
${ }^{24}$ S. Satpathy, A. Roy, and A. Mohapatra, Eur. J. Phys. 33, 863 (2012).

${ }^{25}$ M. Iizawa, S. Kosugi, F. Koike, and Y. Azuma, preprint arXiv:1810.00627 (2018).

${ }^{26}$ M. I. Tribelsky, S. Flach, A. E. Miroshnichenko, A. V. Gorbach, and Y. S. Kivshar, Phys. Rev. Lett. 100, 043903 (2008).

${ }^{27}$ N. Liu, L. Langguth, T. Weiss, J. Kästel, M. Fleischhauer, T. Pfau, and H. Giessen, Nat. Mater. 8, 758 (2009).

${ }^{28}$ F. Hao, Y. Sonnefraud, P. V. Dorpe, S. A. Maier, N. J. Halas, and P. Nordlander, Nano Lett. 8, 3983 (2008).

${ }^{29}$ N. Verellen, Y. Sonnefraud, H. Sobhani, F. Hao, V. V. Moshchalkov, P. V. Dorpe, P. Nordlander, and S. A. Maier, Nano Lett. 9, 1663 (2009).

${ }^{30}$ S. Stassi, A. Chiadò, G. Calafiore, G. Palmara, S. Cabrini, and C. Ricciardi, Sci. Rep. 7, 1065 (2017).

${ }^{31}$ B.-B. Li, Y.-F. Xiao, C.-L. Zou, Y.-C. Liu, X.-F. Jiang, Y.-L. Chen, Y. Li, and Q. Gong, Appl. Phys. Lett. 98, 021116 (2011).

${ }^{32}$ H.-T. Lee and A. W. Poon, Opt. Lett. 29, 5 (2004).

${ }^{33}$ G. Ranjit, D. P. Atherton, J. H. Stutz, M. Cunningham, and A. A. Geraci, Phys. Rev. A 91, 051805 (2015).

${ }^{34}$ G. Ranjit, M. Cunningham, K. Casey, and A. A. Geraci, Phys. Rev. A 93, 053801 (2016)

${ }^{35}$ D. Hempston, J. Vovrosh, M. Toroš, G. Winstone, M. Rashid, and H. Ulbricht, Appl. Phys. Lett. 111, 133111 (2017).

${ }^{36}$ G. Winstone, R. Bennett, M. Rademacher, M. Rashid, S. Buhmann, and H. Ulbricht, Phys. Rev. A 98, 053831 (2018).

${ }^{37}$ R. Diehl, E. Hebestreit, R. Reimann, F. Tebbenjohanns, M. Frimmer, and L. Novotny, Phys. Rev. A 98, 013851 (2018).

${ }^{38}$ A. A. Geraci, S. B. Papp, and J. Kitching, Phys. Rev. Lett. 105, 101101 (2010)

${ }^{39}$ E. Hebestreit, M. Frimmer, R. Reimann, and L. Novotny, Phys. Rev. Lett. 121, 063602 (2018).

${ }^{40}$ M. Toroš, M. Rashid, and H. Ulbricht, Phys. Rev. A 98, 053803 (2018).

${ }^{41} \mathrm{~J}$. Gieseler, B. Deutsch, R. Quidant, and L. Novotny, Phys. Rev. Lett. 109, 103603 (2012).

${ }^{42}$ J. Vovrosh, M. Rashid, D. Hempston, J. Bateman, M. Paternostro, and H. Ulbricht, J. Opt. Soc. Am., B 34, 1421 (2017).

${ }^{43}$ A. Setter, M. Toroš, J. F. Ralph, and H. Ulbricht, Phys. Rev. A 97, 033822 (2018).

${ }^{44}$ M. Rashid, M. Toroš, and H. Ulbricht, Quantum Meas. Quantum Metrol. 4, 17 (2017).

${ }^{45} \mathrm{~J}$. Gieseler, L. Novotny, and R. Quidant, Nat. Phys. 9, 806 (2013).

${ }^{46}$ M. Rashid, M. Toroš, A. Setter, and H. Ulbricht, Phys. Rev. Lett. 121, 253601 (2018).

${ }^{47} \mathrm{C}$. Chatfield, The Analysis of Time Series: An Introduction (Chapman and Hall, 1989), pp. 94-95.

${ }^{48}$ D. Heiman, D. S. Hamilton, and R. W. Hellwarth, Phys. Rev. B 19, 6583 (1979).

${ }^{49}$ M. Rashid, T. Tufarelli, J. Bateman, J. Vovrosh, D. Hempston, M. S. Kim, and H. Ulbricht, Phys. Rev. Lett. 117, 273601 (2016).

${ }^{50}$ D. Goldwater, B. A. Stickler, K. Hornberger, and J. Millen, preprint arXiv:1802.05928 (2018).

${ }^{51}$ M. Iwasaki, T. Yotsuya, T. Naruki, Y. Matsuda, M. Yoneda, and K. Aikawa, preprint arXiv:1812.01230 (2018).

${ }^{52}$ M. Poggio, C. L. Degen, H. J. Mamin, and D. Rugar, Phys. Rev. Lett. 99, 017201 (2007).

${ }^{53}$ D. Kleckner and D. Bouwmeester, Nature 444, 75 (2006).

${ }^{54}$ A. Schliesser, P. Del'Haye, N. Nooshi, K. J. Vahala, and T. J. Kippenberg, Phys. Rev. Lett. 97, 243905 (2006).

${ }^{55}$ P. H. Kim, B. D. Hauer, T. J. Clark, F. Fani Sani, M. R. Freeman, and J. P. Davis, Nat. Commun. 8, 1355 (2017). 